\title{
Ethnologies
}

\section{La « belle salle » du Maramureş}

\section{Anamaria Iuga}

Volume 31, numéro 1, 2009

\section{Passages}

URI : https://id.erudit.org/iderudit/038500ar

DOI : https://doi.org/10.7202/038500ar

Aller au sommaire du numéro

\section{Éditeur(s)}

Association Canadienne d'Ethnologie et de Folklore

ISSN

1481-5974 (imprimé)

1708-0401 (numérique)

Découvrir la revue

\section{Citer cet article}

Iuga, A. (2009). La « belle salle » du Maramureş. Ethnologies, 31(1), 49-76.

https://doi.org/10.7202/038500ar

\section{Résumé de l'article}

Cet article, tiré d'une enquête de terrain, porte sur l'analyse de la « bonne/belle salle " : la pièce où les habitants de la région du Maramureş (Nord de la Roumanie) disposent les objets de la plus grande valeur et qu'ils fabriquent pour la plupart eux-mêmes. Elle est aussi la pièce où les étapes les plus importantes de la vie se déroulent. L'étude se concentre sur d'abord sur l'organisation des objets situés sous la ruda [poutre], et ensuite sur les usages de la salle pendant les rites de passage, surtout pendant les noces telles qu'elles se déroulaient il y a cinquante ans et telles qu'elles se déroulent toujours à l'heure actuelle. La « belle salle » du Maramureş, par son organisation et ses usages, est un bon exemple de la dynamique de la culture locale où le présent et les innovations côtoient les pratiques transmises par la tradition.
Ce document est protégé par la loi sur le droit d'auteur. L'utilisation des services d'Érudit (y compris la reproduction) est assujettie à sa politique d'utilisation que vous pouvez consulter en ligne.

https://apropos.erudit.org/fr/usagers/politique-dutilisation/ 


\section{LA « BeLle SALLE » DU MARAMureş}

Anamaria IUGA

Musée du paysan romain, Bucarest, Roumanie

Présente dans presque toutes les maisons de villages roumains, la pièce où les habitants mettent les objets de la plus haute valeur, la plupart fabriqués de leurs mains, est l'espace festif par excellence, espace qui se trouve à l'écart du quotidien. Dans cette salle sont exposés des objets chargés d'un sens particulier : meubles, tapis, couvertures, serviettes, des coussins avec des taies d'oreillers brodées à la main ou tissées, les plus belles icônes, des assiettes en émail. Cette pièce contient également la dot de la fille qui est soit placée dans des coffres, soit exposée sur les murs. Les événements les plus importants de la vie des gens se passent à l'intérieur de cette pièce, surtout ceux qui ont trait à certains " rites de passage " (Van Gennep 1996); c'est là que sont reçus les invités lors de noces ou de baptêmes; une bonne partie de la noce se passe encore dans cet espace, et c'est également dans cette pièce que l'on se retrouve après les enterrements. Quelles que soient les occasions, cette salle doit être toujours propre et prête à recevoir les gens de passage. Cet espace apparaît donc comme le lieu où se déroulent un ensemble de rites de passage et d'agrégation qui structurent les relations et les rapports sociaux. 
Dans la région du Maramureş' en Roumanie, cette salle porte plusieurs noms qui expriment tous la primauté de cette pièce sur les autres au travers de dénominations telles que "belle salle" ou "bonne salle [camera bunã] " / "belle/bonne maison [casa bunã] ». "La salle de devant [camera dinainte] " et "la grande maison [casa mare] ", ou d'autres encore qui expriment l'importance de cette salle par ses dimensions. "La salle avec la "ruda " [ camera/casa cu rudã] ", une dénomination qui met l'accent sur un ancien élément de décoration de la maison rurale traditionnelle - la culme, appelée la ruda [poutre] dans le Maramureş, soit une poutre suspendue, d'un diamètre de dix à douze centimètres, qui longe le mur le plus long de la salle et sur laquelle on accroche des tissus dans un ordre spécifique.

On la nomme encore "la salle de fêtes" et de "la parade [camera de sãrbãtori ; camera de parade] ", insistant alors sur le fait que cette pièce est utilisée pour des activités collectives et est le lieu d'exposition du privée au public.

Un autre nom utilisé depuis plus récemment est "la salle "habillée à la mode paysanne" [camera "îmbrãcatã tãrãneşte"] ", ou bien "la salle "paysanne" [ camera "tãrãneşte"] " tout court, qui exprime une certaine attitude duale qui définit le monde rural contemporain : d'une part, il y a l'attitude "traditionnelle", selon laquelle la pièce est un prolongement de l'être humain et qui a donc besoin "d'être habillée ", comme tout objet présent dans l'espace villageois; d'autre part il y a l'attitude "moderne", qui fait que les sujets ont un regard extérieur sur eux-mêmes, créant ainsi un métadiscours, en se construisant euxmêmes comme des paysans, face aux autres personnes qui visitent leur maison.

1. Je remercie Antoine Heemeryck pour sa correction d'une version antérieure de ce texte et pour ses conseils nombreux. Je reste seule responsable du contenu de ce texte.

Le Maramureş [se lit "Maramurech"] est un département situé dans le Nordouest de la Roumanie, divisé en quatre régions différentes du point de vue folklorique et ethnographique, traditionnellement surnommés "pays ": "le pays de Lãpuş» dans la partie de sud-est, "le pays de Codru " dans la partie de sud-ouest, "le pays de Chioar» dans la partie centrale-sud, et "le pays de 
Cet article, tiré d'une enquête de terrain ${ }^{2}$ dans deux villages de la région du Maramureş, porte sur l'analyse de cette "bonne salle », ses fonctions et usages sociaux, mais aussi sur son organisation, en essayant de comprendre la dynamique du monde rural contemporain. Dans cette étude, je vais me concentrer sur la position de la «belle salle " dans l'ensemble de la maison et plus précisément, sur un seul élément, la ruda [poutre suspendue] - sa position dans la «belle pièce ». L'analyse principale cible notamment l'organisation et la dynamique entourant les objets qui se trouvent sous cet élément de décoration, mais aussi sur les usages de la salle pendant les rites de passage, en particulier, les noces.

L'analyse est fondée, d'un côté, sur des sources historiques: la documentation de musées et les descriptions tirées de livres d'ethnographie classique, et surtout sur des entretiens menés avec les acteurs locaux, ainsi que sur des observations directes de l'utilisation actuelle de la "belle salle».

Maramureş" dans la partie du nord. Ce dernier "pays » est aussi nommé "Maramureş historique ", et donne son nom à tout le département. La région ethnographique du Maramureş est délimitée au nord et nord-est par les Montagnes de Maramureş (1500-2000 m), dans le sud-est par les Montagnes Rodnei $(2000 \mathrm{~m})$ et dans le sud par les Montagnes Tibleş-Oaş-Gutâi (1000$1400 \mathrm{~m}$ ). Dans le Nord, sur $60 \mathrm{~km}$, se trouve la rivière Tisa, qui représente aussi la frontière avec l'Ukraine, dont fait parti les deux-tiers de l'ancien « Maramureş historique » comme cela est attesté dans des documents, à partir du XIV“ siècle jusqu'en 1920 (Filipaşcu : 1945).

2. La recherche de terrain a débuté en 2001 et s'est poursuivie pendant l'hiver 2004-2005 et l'hiver 2005-2006. C'est une recherche fondée sur des méthodes interprétatives, mettant l'accent sur la manière dont les sujets comprennent et perçoivent le rôle et l'organisation de la « belle salle ". J'ai utilisé des techniques qualitatives, comme l'entretien semi directif, et l'observation participante. Pour trouver les maisons où il y a des salles "habillées à la mode paysanne ", je me suis laissée porter par les conseils des gens des villages pendant les entretiens et lors de rencontres dans la rue. De cette manière j'ai pensé trouver les « belles salles " que ces gens considèrent représentatives pour la communauté. En tout, j'ai visité dix-sept maisons à Saliştea de Sus et j'ai fait douze entretiens semi directifs; et j'ai vu sept maisons à leud et ai conduit quatre entretiens semi directifs. 
Cette recherche a été réalisée pendant les années 2001, 2004-2005 et 2006, dans la région appelée "pays de Maramureş" ou "Maramureş historique " qui est divisée, du point de vue des pratiques culturelles, en sous-régions, selon " les particularités des phénomènes culturels et artistiques" (Zderciuc 1963: 7). Cette division est présente aussi dans l'organisation de la "belle salle ", par des particularités qui peuvent être observées dans les villages situés aux abords des rivières qui traversent le département : les rivières de Mara, Iza, Cosãu et Vişeu. Les deux localités qui font l'objet de la présente recherche : Sãliştea de Sus (en 2002: 5482 habitants) et Ieud (en 2002: 4442 habitants), sont situées dans la Vallée de l'Iza. Les règles d'organisation de la " belle pièce " sont similaires dans les deux localités, malgré certaines différences, ce qui souligne l'importance pour les gens d'affirmer une identité différenciée, par rapport aux membres d'une autre communauté locale.

La «belle pièce " dans le Maramureş, témoigne d'une société traditionnelle et moderne à la fois : c'est à dire dont les habitants suivent les règles de vie transmises à travers les générations, et qui, dans le même temps, vivent dans un monde en changement constant et où apparaissent de nouvelles valeurs. C'est un monde qui est mis sous le signe du passage, un passage à un monde mélangé, dont les anciennes façons de vivre coexistent avec les nouvelles. Les habitants des villages, qui appartiennent à un milieu social régi selon d' " anciennes " règles de vie, parfois "réinventées" (Hobsbawm \& Rangers), vont travailler dans d'autres régions, et surtout dans d'autres pays, pour revenir ensuite

3. Les principales activités de travail des acteurs dans les deux villages sont l'agriculture et l'élevage des animaux. Pendant la période communiste, Sãliştea de Sus et leud ont été collectivisés, ce qui a imposé des changements sociaux d'une grande violence. La systématisation a eu lieu non pas parce qu'il y avait un riche terrain agricole, mais pour détruire, comme l'idéologie le demandait, la classe des paysans possesseurs de terres, les nemeşi, descendants des familles paysannes nobles de la région (Kligman 1998:26). Une autre raison, qui explique cette attaque de l'Etat-parti, est le poids de l'Eglise gréco-catholique dans les agencements micro-politiques et microsociaux, qui, après 1989, a repris son rang d'autorité (Kligman 1998: 26). La collectivisation créa dans cette région de larges modifications économiques, sociales, culturelles et politiques. La société rurale se trouva face à des impositions politiques violentes. Après la chute du communisme, les gens ont repris leurs terres, et avec elles, ils se sont réapproprié un mode de vie inspiré par des pratiques anciennes, qui se sont insérées, avec d'autres règles sociales, dans les changements qui portent vers une société moderne. 
dans leur lieu de résidence avec un peu d'argent. Cette migration souligne les problèmes liés à la subsistance et à la possession d'argent. Ce phénomène entraîne des changements qui vont se refléter dans les choix que ces gens font tous les jours et ce, même dans le choix d'avoir une ruda dans la "belle salle». La "pièce de devant" est décorée avec des objets faits à la main par les femmes au foyer, et au travers lesquels les gens des deux localités expriment leur attachement aux règles communautaires. Mais, en même temps, il y a là des changements visibles dans l'organisation et dans l'ornementation des objets qui nous éloignent d'une interprétation en termes d'ancestralité et de pureté "des habitudes paysannes".

\section{De belles malsons avec de "belles salles"}

La maison et ses alentours ont toujours été le centre de la vie sociale, culturelle et économique des individus. L'habitat domestique est une " unité sociale complexe", un "phénomène total de vie sociale " (Cristescu-Golopentia 2002 : 34) qui, de par sa signification, est une des instances de socialisation primordiale liée à la dynamique complexe de la définition de soi en tant qu'acteur appartenant à un groupe social et culturel donné. La manière dont les agents sociaux construisent les maisons (individuellement ou collectivement, avec la famille, etc.), la façon dont ils occupent l'espace, la signification qu'ils lui donnent et la façon dont ils utilisent, produisent et donnent une signification aux objets (selon l'âge, le sexe et le statut social), tout cela est révélateur de la culture dans laquelle ils vivent et qu'ils produisent : une culture en pleine transformation, mais aussi conservatrice par certains aspects.

Pour comprendre ce milieu et les influences que subit la «belle salle ", il faut s'appuyer sur l'observation de la maison. Il faut spécifier que la maison est visible de l'extérieur, ce qui, dans le cadre d'une logique d'économie de prestige, facilite l'expression du statut social au public, à la différence de l'intérieur de la maison qui relève du privé.

Gavrilã H., de leud, 67 ans, explique ceci selon un principe méritocratique (selon lequel les acteurs plus importants et les plus méritants par leur travail dans la communauté étaient aussi les plus riches) :

Ceux qui étaient pauvres n'avaient pas [deux pièces dans la maison], au contraire, ceux qui avaient une position matérielle meilleure, qui étaient de bons fermiers, ils avaient deux pièces, une entrée et un office (Gavrilã H. 2005). 
Les maisons en bois constituées de deux pièces, une entrée et un office pour les alim en ts, portaien $t$ le nom de " $m$ aison des nemeşi [casã nemeşeascã] "(Nistor 1980: 13), marquant ainsi la position sociale élevée de ces personnes dans la communauté, car les nemeşi, les nobles paysans (ordre social qui a été quasiment détruit pendant la période communiste) étaient aussi les plus riches du village. À l'époque, dans la hiérarchie de la communauté, il y avait une corrélation entre la position sociale et le pouvoir économique.

Sous l'impulsion du communisme et après sa chute et l'intégration de structures capitalistes, l'organisation sociale a changé. L'architecture de la maison a également changé. Les nouvelles maisons à étages, les maisons en briques sont devenues de plus en plus fréquentes dans les localités de la région, dès les années 1970. Comme à Ieud et à Sãliştea de Sus, les terres ont été collectivisées et le premier aspect de la propriété privée (avec les terres), sur laquelle s'appuyait la hiérarchisation entre acteurs, était précisément la maison. Une des raisons pour laquelle les maisons à étages ont commencé à être construites dans les années 1970. 1980, s'explique par le fait que les nouvelles normes d'architecture communistes l'imposaient (dans les villages il était interdit de bâtir des maisons avec un seul niveau), fait qui est aussi mentionné par Gail Kligman (1998). Or, une autre explication est offerte par les gens de la communauté :

Probablement qu'ils ont vu de belles maisons où ils sont partis [travailler]. Ils sont rentrés avec de l'argent et, comme il n'était plus possible d'acheter des terres ou trop d'animaux, ils ont investi cet argent dans une belle maison (Simion I. 2001).

Aujourd'hui, quelques-uns regrettent d'avoir construit une maison en brique, pour des raisons pratiques : à la question de savoir s'ils n'auraient pas préféré une maison en bois à l'instar de nombreuses personnes, Anuta I. le souligne: "Si j'avais su qu'elle serait si froide, j'aurais construit [une maison] en bois " (Anuta I. 2005).

Le fait est qu'il faut avoir une maison et, selon les croyances populaires, qui sont aussi l'expression symbolique des normes sociales, dans sa vie, un homme doit planter un arbre et également construire une maison (Vlad 2002: 473). Pour les habitants des deux localités, il est bien connu que lors d'un mariage, la maison est traditionnellement la contribution des garçons, mais, depuis récemment, cela n'est plus systématique, comme explique Maria V., 52 ans: 
Avant c'était comme ça : le garçon apportait la maison et la fille la dot. Avant c'était comme ça. Par exemple, mon mari n'avait pas de maison, et mon père n'a pas accepté ça. Si à 25 ans il n'a toujours pas de maison, ce n'est pas un homme.... Maintenant, vous savez, il y a peu d'enfants dans une famille ... et dans de nombreux cas les deux mariés ont chacun une maison et vont là où ils veulent. Mais normalement c'est le garçon qui doit en avoir une ... et ceux qui sont paysans font tout pour avoir une maison. Les filles ne veulent pas aller vivre avec les belles-mères. Elles veulent avoir leur maison, être indépendantes (Maria V. 2005).

Les maisons visitées pendant notre recherche de terrain représentent presque toutes les types de constructions qui peuvent être rencontrées dans la région rurale du Maramureş. Toutes ces maisons avaient une "belle salle " dans un autre endroit dans la maison. Dans les lignes suivantes je vais illustrer les différents types de constructions avec les maisons les plus représentatives.

Dans les anciennes maisons, comme dans les maisons de la région du Maramureş observables dans les Musées de villages de Baia Mare et de Bucarest (où il y a même une maison de leud), lorsqu'elles comprenaient deux pièces et une entrée, la "belle salle " constituait la plus grande pièce, parce qu'elle était le plus souvent utilisée pour les réunions de famille et il était donc nécessaire d'avoir beaucoup d'espace pour que tous puissent y loger. La localisation de la "salle de devant » dépendait de la position de la maison par rapport à la rue et aux points cardinaux (l'arrière de la maison est traditionnellement orientée vers le Nord): La salle festive doit normalement être "dans la partie plus obscure ", comme Maria C. (50 ans, Sãliştea de Sus) l'explique.

La chambre où le propriétaire passe la plupart de son temps doit être orientée vers la rue, puisque le paysan aime voir et communiquer, voir ce que se passe dans la rue, qui passe, qui arrive (Maria C. 2006).

Dans les deux localités, je suis également entrée dans quelquesunes des anciennes maisons en bois. À Ieud la maison d'Irina C. (75 ans, leud), en était un bon exemple. Elle y vivait avec son mari, Toader, et une de ses filles, Anuta. Ils étaient la quatrième génération à vivre dans cette maison:

Le grand-père de mon père, Toader Vãlenari, c'est lui qui a construit la maison. Je suis au quatrième rang des hommes qui y vivent (Toader C. 2005). 
Selon eux, cette maison a plus de 180 ans. Elle occupe un périmètre d'environ quatorze mètres de long et sept mètres de large. La "salle de devant " est de sept mètres carrés. Elle est décrite par les informateurs comme "la grande maison". La porte était fermée à clé, preuve qu'on y entrait rarement. Les petits-fils d'Irina, par exemple, alors en visite, n'ont pas eu le droit d'y entrer, alors que je prenais des photos de cette pièce.

Dans les deux localités, on peut observer aussi des maisons en brique et en béton qui suivent en partie les règles dites traditionnelles d'organisation de l'espace et de délimitation des pièces. Un exemple de maison de ce type est la maison de Mãricuta C. de Ieud, 36 ans, qui vit là avec son mari (qui part la plupart de l'année travailler en Italie) et ses cinq enfants. Dans sa maison, construite entre 1988 et 1990, il y a trois pièces, une entrée et un office. $\mathrm{La}$ "grande salle " est de cinq mètres sur quatre et est située dans la partie droite ; on y accède depuis l'entrée.

La maison qui fait la transition entre maison à un seul niveau et maison à étage est la maison de Gavrilã $\mathrm{H}$. de leud, 67 ans et de sa femme, Ioana H., 62 ans. Celui-ci est sculpteur sur bois et restaurateur d'église et de maison en bois et est très connu en Roumanie. Dans sa famille, comme il l'explique, il est la troisième génération de sculpteurs de bois, métier qu'il a également appris à ses quatre fils. Il a travaillé pendant plusieurs années au Musée du Maramureş, à Sighetu Marmatiei, mais est à présent retraité. Dans sa résidence domestique, il y a deux maisons en bois. Une a été construite en 1912 : elle comporte une entrée et deux pièces; c'est là où vit la famille de son plus jeune fils (quatre personnes). L'autre maison a été construite en 1981 et un étage y a été ajouté il y a quatre ans. Cette fois, la " salle avec la ruda" est la plus petite pièce de la maison; elle fait deux mètres sur deux mètre cinquante, et se trouve à l'étage.

Les autres maisons sont toutes en brique et s'étendent sur deux niveaux. La "pièce " paysanne " ", dans ce dernier cas — les maisons à étage --, est uniquement décorative et est normalement située à l'étage. C'est le premier pas vers sa disparition, comme chez Florica P. de Sãliştea de Sus, 42 ans, qui vit dans une maison bâtie en 1984, sur deux niveaux, où, même s'il y a des textiles accrochés dans la maison, ils ne sont pas exposés dans une salle spéciale, il n'y a plus de "belle salle", comme expliqué au début. 
Au moment de choisir les maisons pour analyser la "salle "habillée à la mode paysanne" ", j'ai essayé de voir diverses maisons qui puissent donner une image assez complète de la constitution de la "belle salle " et de sa localisation dans la maison. Il apparaît en effet que la "belle salle"se trouve dans des endroits différents selon les maisons. Pour les maisons n'ayant qu'un rez-de-chaussée, le plus fréquent est d'avoir la «belle salle " à l'opposé de la rue. Il y a une délimitation spatiale entre festif et quotidien qui s'opère sur le plan horizontal. Pour les maisons à étage, cette division de l'espace physique peut être mise en valeur dans une analyse verticale de l'espace. Dans tous les cas, sauf celui de Ileana V. de Sãliştea de Sus, la « belle salle » est située à l'étage. Nous pourrions donc établir que les activités quotidiennes ont lieu au rez-de-chaussée pour des raisons pratiques, et que, pendant les fêtes et célébrations familiales, quand l'espace quotidien ne peut pas se transformer en espace festif, l'étage revêt alors cette fonction. Pour les gens de la région de Maramureş l'espace où il est traditionnellement possible de vivre le festif, est la "belle pièce ". Il est donc évident que si la distinction " quotidien / festif » est représentée de manière verticale ; c'est une salle de l'étage qui doit être "habillée ", selon les règles d'organisation " traditionnelles", et être ainsi nommée « la belle salle».

\section{" Habiller " la " belle salle "}

La "belle salle " passe d'une pièce à l'autre dans la maison. Aussi, les objets qui se trouvent dans les maisons changent d'une forme et sont placés à d'autres endroits, car il est évident que l'intérieur d'une maison " ne peut jamais être considéré comme fini, mais est un milieu soumis à une évolution permanente » (Stoica et Doagã 1977 : 12). En premier lieu, il faut remarquer que dans la « salle de devant », la plupart des objets sont des textiles. Cette pièce est décorée surtout avec des tissus, qui sont tous faits dans la maison, par la mère de famille et ses filles, en conformité avec les normes de la communauté :

Tous sont faits dans la maison; il n'est pas question d'acheter un tapis.

C'est même une honte d'aller acheter [des tissus] (Viorica I. 2001).

Comme nous le montrerons, l'association des objets et leur agencement sont déterminés par " un schéma constant, d'un caractère stéréotypé », qui suit quelques principes (Bobu Florescu et Petrescu 1969 : 183). Je vais à présent analyser les objets qui sont présentés sous la ruda de la "belle salle », et de voir quels sont les objets qui respectent 
ce "schéma constant", soit les objets que je nomme "objets forts", et quels sont les objets qui ne le respectent plus et qui témoignent d'une évolution permanente. Je vais nommer " objets faibles" les objets qui opèrent le passage vers une autre forme d'organisation, et qui vont probablement au fil du temps établir une autre «tradition » en devenant des objets de pratiques partagées.

\section{La ruda}

Dès l'entrée dans une "belle salle " traditionnelle, le coin avec le plus d'effet est le mur avec la ruda et les lits (Zderciuc 1963: 17). La ruda est un ancien élément de décoration de l'intérieur paysan, nommé culme dans d'autres régions, et qui, au cours du XIX ' siècle a été généralisé dans les régions de Moldavie, du Maramureş, dans le Nord-ouest de la Transylvanie, au Banat et en Hunedoara (Zderciuc, Petrescu et Bãnãşeanu 1964 : 14).

La ruda est donc une poutre suspendue au plafond, longeant le mur le plus long de la pièce - le mur "de derrière " - sur laquelle on agence dans un ordre spécifique diverses catégories de tissus. C'est sur cette poutre qu'est aussi exposée la dot des filles. La ruda est ainsi vue comme " un des signes extérieurs les plus visibles de la situation matérielle et de la position sociale des habitants de la maison " (Zderciuc 1963 : 15) et, dans le même temps, c'est une offre, comme sur un marché, dans la circulation des femmes-filles.

Tel que signalé par Boris Zderciuc (1963), la ruda connaît des variantes en fonction des sous-régions de la région du Maramureş. Ieud et Sãliştea de Sus sont deux localités situées dans la même vallée: la Vallée d'Iza. Les règles générales d'organisation y sont similaires malgré quelques variations. Il y a pourtant des différences dans l'organisation, qui sont le signe d'une volonté d'exprimer des particularités et une appartenance à une communauté spécifique.

A Ieud (voir photo no.1), la ruda contient quatre couches d'objets. La première couche est constituée de couvertures appelées lati. Ce sont des couvertures en laine, tissées avec des fils de laine plus gros du type des couvertures qui se mettent sur les lits et qui sont tissées selon la même technique, connue sous le nom de cergã. Ces couvertures spécialement faites pour la ruda, ont des motifs géométriques qui sont 


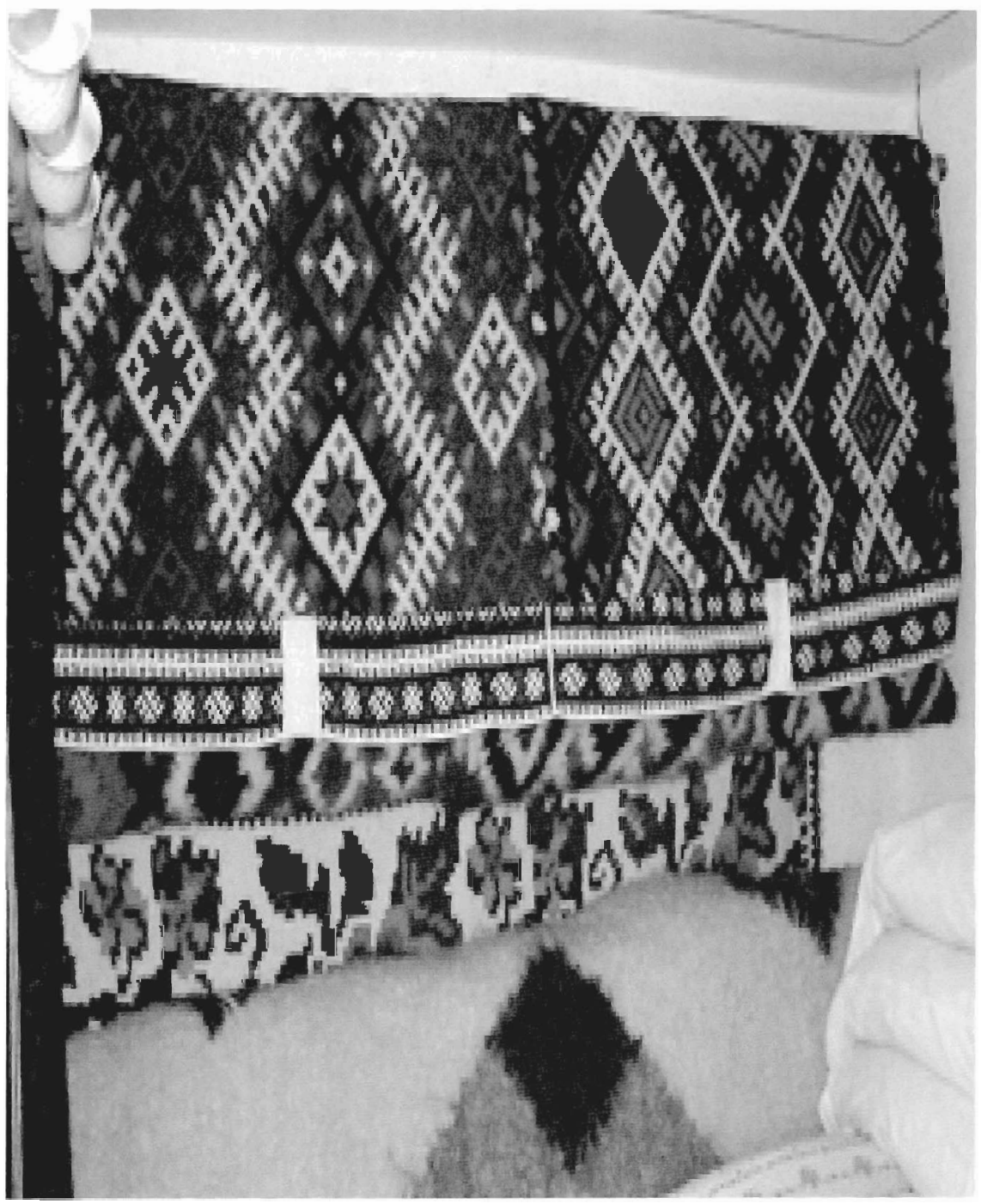

1. La ruda de leud (A. luga, 2004). 
appelés crãngi dans le village. Les lati sont placées en largeur, elles se plient et une des extrémités est placée par-dessus la poutre. Pour cette raison il faut avoir plusieurs lati pour couvrir la poutre en fonction de la longueur du mur. Pour la ruda d'Irina C., 75 ans, longue de sept mètres, il a été nécessaire d'y accrocher cinq lati. Pour la ruda d'Anuta D., 23 ans, longue de quatre mètres, il en a fallu quatre, dont une a été repliée davantage par manque d'espace. Chez Gavrilã H., 67 ans, il y a seulement deux lati, et chez Mãricuta C., 36 ans, il y a quatre lati. Partout où je suis allée j'ai constaté la présence de lati avec des motifs géométriques, donc c'est un des objets forts qui font partie de la pratique partagée et ne changent ni de forme, ni de position.

Au-dessus il faut mettre des nappes, nommées fãtoi, qui sont réalisées à base de chanvre au métier à tisser, et qui comportent des motifs dans la partie inférieure, la seule partie visible. Il est nécessaire, dans ce cas aussi, de fabriquer plusieurs draps, qui sont ensuite unis par de la dentelle faite à la main ou, c'est la cas depuis récemment, achetée. La partie inférieure de ces nappes peut être ornée avec de la dentelle appelée colti et qui peut également être achetée. Le seul endroit où il n'y avait pas de dentelle dans la partie inférieure, c'était chez Gavrilã H., mais par contre, chez Anuta D., la dentelle était aussi grande que les ornements. Les nappes sont les premiers éléments de la ruda qui opèrent le passage vers une autre règle: ce sont des objets forts, par rapport à leur position et leur présence sur la poutre, mais, comme il est possible de le voir, les ornements changent de composition, car sur le même drap on peut voir apparaître des ornements géométriques et floraux, comme dans le cas de Mãricuta C., et il peut y avoir ou non de la dentelle ou de la broderie.

Sur les nappes il faut mettre les tapis, toujours en largeur. Les tapis de ruda sont considérés comme les plus beaux et plus importants tapis de la maison. Ils sont faits spécialement pour décorer la ruda. Dans certains villages ils sont nommés rudas (dénomination que l'on retrouve aussi à leud: Mãricuta C., parlant de son tapis sur la ruda, a utilisé ce terme). Traditionnellement, dans la vallée d'Iza, le tapis utilisé pour orner la ruda est disposé en largeur, plié en deux au milieu, une des parties étant placée sous la poutre. Il est nécessaire de mettre plusieurs tapis pour couvrir toute la poutre : ainsi, pour la ruda d'Irina C., il a fallu utiliser cinq tapis, et pour la ruda de Gavrilã $\mathrm{H}$., seulement deux.

Dans ce village les tapis utilisés pour la ruda ont des motifs géométriques. Comme explique Gavrilã H., si quelqu'un veut faire un 
tapis pour la ruda et qu'il n'a pas de modèles d'ornements, il doit aller à l'église où se trouve une belle collection de tapis qui respectent la spécificité locale pour s'en inspirer, plaçant ainsi les acteurs dans un processus de légitimisation par le respect de la tradition :

Il est de coutume dans le village que si un membre de la famille décède, le père ou la mère, l'on place une icône et un tapis sur le cercueil. Au fil des années, vous vous rendez compte combien de tapis il y a dans nos églises. ... Et qu'est-ce que les femmes font? Si elles ne trouvent pas de modèle ailleurs, elles prennent des motifs d'anciens tapis de l'église. ... Ma sœur, par exemple, avant de se marier, il y a cinquante ans, elle n'avait aucun modèle, je ne l'ai jamais oublié, et mon père, qui avait la ćlé de l'église dans la Plaine, y est allé et a porté un tapis de l'église pour que ma sœur prenne le modèle (Gavrilã H. 2005).

Gavrilã $\mathrm{H}$. donne également l'exemple de sa femme qui a vendu sa ruda et sa dot. Il explique:

Voilà comment Ioana, ma femme a fait : elle a fair une copie de la dor qu'elle a reçue de sa mère, et qu'elle avait vendue au musée de Sighet. Immédiatement elle s'est rendu compte, grâce à moi, qu'elle avait fait une erreur, mais elle en a fait une copie, avec les mêmes modèles, les mêmes ornements, en prenant des tapis de l'église du village (Gavrilã H. 2005).

Sur la ruda, tout en haut, tous disent que l'on met des oreillers longs, nommées cãpãtâie, avec des ornements qui sont placés à une seule extrémité de l'oreiller. Des taies d'oreiller sont également réalisées au métier à tisser, et cette fois elles ont des ornements floraux. Mais dans des situations que j'ai pu observées, les oreillers n'étaient pas mis sur la ruda: ils étaient "descendus " et disposés sur le lit, ce qui nous porte à croire qu'il faut considérer les oreillers comme des objets faibles.

À Sãliştea de Sus (voir photo no. 2), la ruda se trouve toujours sur le mur le plus long de la salle et, dans quelques cas, sur la moitié du mur perpendiculaire, comme il m'a été permis de constater dans quelques maisons (chez Maria I., 65 ans, Maria Ch., 46 ans, et Maria C., 50 ans).

Dans cette ville, la ruda comporte six couches. Les couvertures forment la première couche. Ici, elles portent le même nom que pour les couvertures qui se mettent sur les lits : cergã. Ces couvertures ont aussi des ornements. Traditionnellement, il y avait, comme à leud, des ornements géométriques, mais que je n'ai trouvés que dans le cas de 


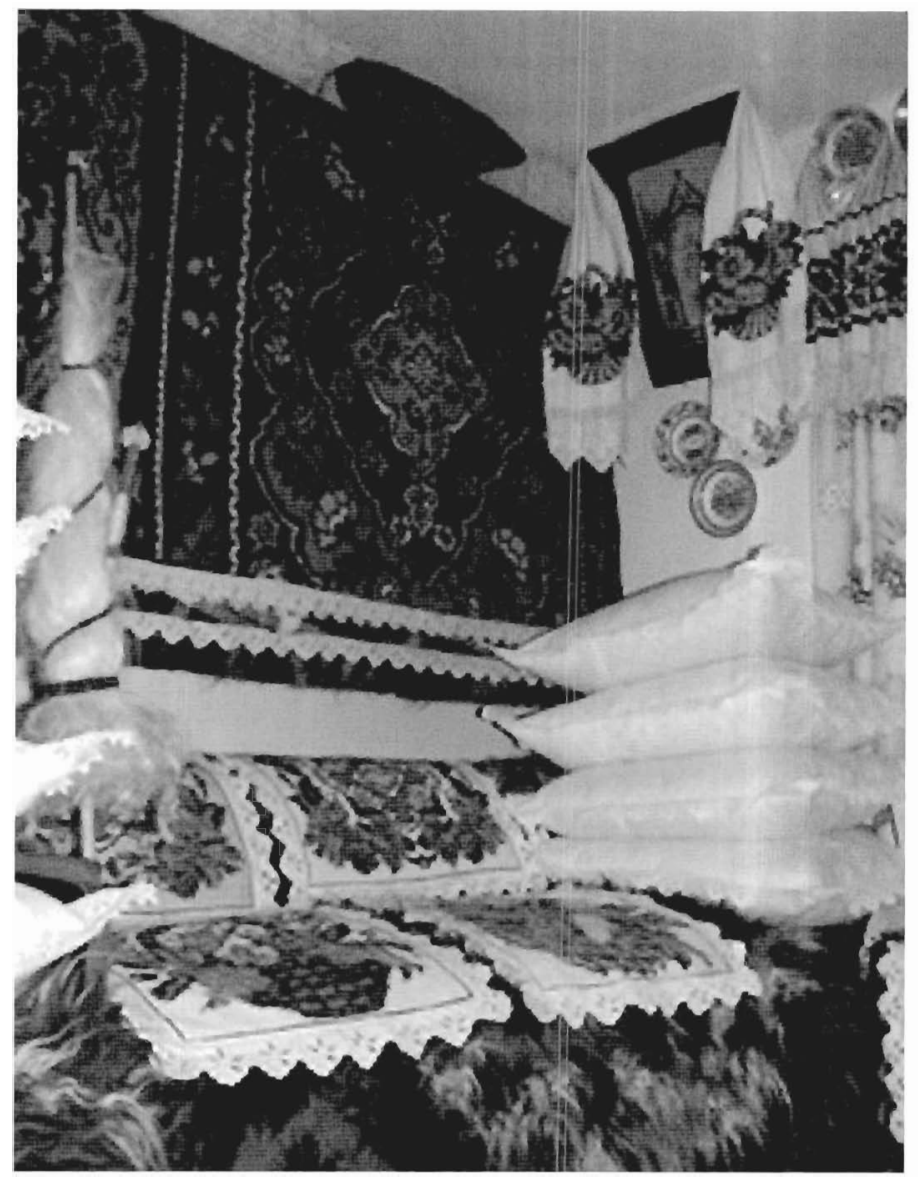

2. Une ruda à Sãliştea de Sus (A. luga, 2000).

Maria V., 52 ans (voir photo no. 3). C'est un cas spécifique, puisqu'elle est originaire de Dragomiresti, le village voisin, et qu'elle s'est mariée à Sãliştea de Sus et a organisé sa "belle salle " comme dans son village natal. Pour cette raison, et du fait des intentions de Maria V., cette "salle avec la ruda" est particulière, car elle est toute ornée de tissus qui comportent seulement des motifs géométriques. Dans toutes les autres maisons à Sãliştea de Sus, selon la tradition locale, la couverture a des modèles floraux; elle se voit très peu, parce qu'elle est couverte par les nappes qui composent la couche suivante. Du point de vue de leur présence sur la ruda, les couvertures constituent des objets forts. Les couvertures deviennent objets forts par rapport aux ornements, 


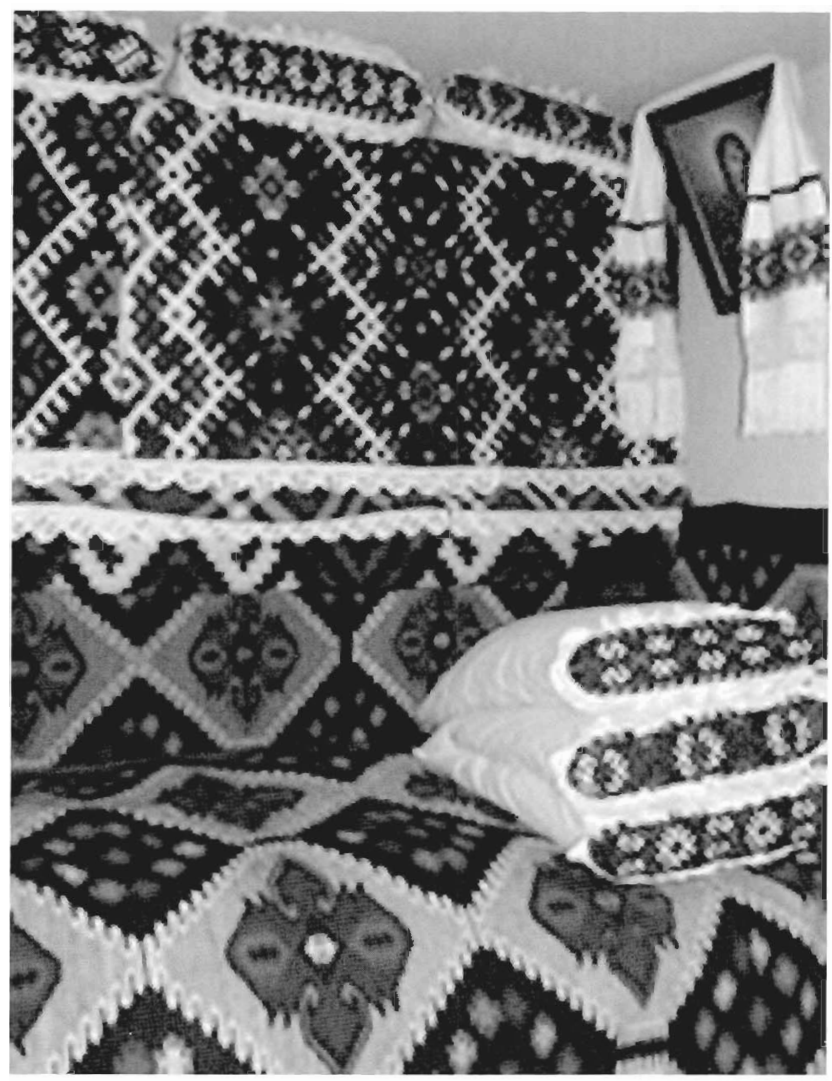

\section{Couvertures chez Maria V. (A. Iuga, 2005).}

parce que les modèles floraux sont considérés comme traditionnels par les sujets, et c'est ce qu'ils les montrent pour exemplifier leur tradition. Comme à leud, on dispose la couverture en longueur, puisqu'il faut mettre sur la ruda plusieurs cergi (chez Maria V., il y avait trois couvertures; chez Ileana V., aussi trois couvertures; chez Maria I. il y avait quatre couvertures, etc.).

Au-dessus des couvertures, il faut mettre les nappes, nommées aussi fãtoi et lepedeauã, et qui sont faites à base de chanvre, au métier à tisser, et qui comportent des motifs dans la partie inférieure et dans quelques cas des broderies (comme chez lleana V., Maria V., Anisia L. et Maria Ch.). Comme à Ieud, elles sont tissées et étroites, et il faut qu'elles 
soient liées entre elles par de la dentelle. Les ornements des nappes sont composés de motifs floraux, à l'exception bien sûr de la ruda de Maria V. Dans certains cas, les nappes de cette couche n'ont pas d'ornements, comme chez Mãricuta V. et Maria I., où il s'agit de nappes simples. Comme dans le cas des couvertures, il est possible de les considérer comme des objets forts, de point de vue de leur présence et de la façon dont elles sont ornées.

La couche suivante est composée d'une toile de laine, mise en longueur, avec des ornements en forme de carreaux ; cette toile est la même que celle utilisée pour les besaces [desagi], elle est d'ailleurs appelée desagi. Dans le Maramureş, il y a des besaces pour tous les jours; certaines comportent des carreaux, combinant seulement le blanc et le noir, d'autres sont réservées aux jours de fêtes et combinent le blanc, le noir et le rouge. Chaque village a son propre modèle de besaces. Ainsi il est possible de différencier les gens entre eux et d'établir quel est leur village d'origine, comme l'expliquaient Simion I. (avril 2001) et Viorica I. (avril 2001). Sur la ruda les gens mettent seulement la toile de besaces de fête, qui, à Sãliştea de Sus, comporte des carreaux plus grands que celle de Dragomireşti et que Maria V. a mis sur sa ruda. Cette couche est composée d'un objet fort: la toile de besaces, qui ne change ni de forme, ni de composition. Au-dessus il faut mettre de nouveau des nappes, fãtoi, qui ont les spécificités des nappes de la deuxième couche.

Dans les maisons où résidaient des personnes âgées (Maria I., 65 ans, et Ion Simion V., 74 ans) et dans le cas de Anisia L., 51 ans, la ruda n'a pas ces deux dernières couches (toile de besaces et une autre couche de nappes) : elle est identique à la ruda de Ieud, avec quatre couches.

Sur toutes ces couches, on met les tapis. De même qu'à leud, le tapis pour la ruda se place en largeur, plié en deux au milieu, une des parties étant mise sous la poutre. Il est nécessaire de mettre plusieurs tapis pour couvrir toute la poutre : ainsi, pour la ruda de Maria V., il y avait trois tapis; chez Ileana V., aussi trois tapis; chez Maria I., il y avait quatre tapis ; chez Mãricupa V. il y avait toujours trois tapis ; chez Maria Ch, il y avait quatre tapis; Anisia L. avait trois tapis et lon Simion V. avait trois tapis.

En ce qui concerne les ornements à Sãliştea de Sus, il est possible de comprendre leur fonctionnement à différentes étapes. Chez Maria V., qui est née à Dragomireşti et qui a fait sa ruda comme cela se fait dans son village, les tapis ont des ornements géométriques. Ce type 
d'ornements est celui qui se faisait aussi anciennement à Sãliştea de Sus. Dans les maisons où vivaient des personnes âgées, chez Maria I. et chez Ion Simion V., les tapis sont dans une étape transitoire, qui combine des ornements floraux avec des formes géométriques, et, dans quelques cas, on trouve même des fleurs de forme géométrique. Selon les villageois, la dernière étape, plus récente, a commencé il y a vingt ans (Simion I. 2001). Maintenant les tapis ont des ornements floraux très naturalistes, ce qui donne le caractère spécifique local. Comme Maria V. l'explique, elle a fait sa "pièce "habillée à la mode paysanne" " et la ruda comme cela se fait dans son village: seulement avec des tissus à ornements géométriques. Elle dit que les gens viennent voir sa "chambre", pour comparer avec la ruda de Sãliştea:

Les gens viennent aussi par curiosité, pour comparer Sãliştea à Dragomirepti... Par exemple, ma belle-sœur qui est ma voisine, a la ruda, mais comme elle se fait en Sãliştea, bien sûr, avec des fleurs. Et il y a des personnes qui viennent et comparent (Maria V. 2005).

Dans cette situation, quand les règles anciennes d'ornementation sont abandonnées et les nouveaux ornements sont considérés emblématiques pour la localité, le tapis est passé d'objet faible est devenu un objet fort.

En haut sur la ruda, il faut, aussi, mettre les oreillers longs, nommés cãpãtâie, dont les taies d'oreiller ne comportent des ornements qu'à une seule extrémité, et qui sont placés de telle sorte que ces ornements se voient. La toile des oreillers est faite au métier à tisser, et les extrémités sont brodées à la main avec des ornements floraux. Dans les dernières années, est apparue la mode de broder ces oreillers blancs avec des ornements blancs ou de mettre au lieu de la broderie en couleurs de la dentelle blanche.

Sur la ruda les femmes mettent des oreillers longs brodés de fleurs en couleurs et, si possible dans les mêmes nuances que le tapis qui se trouve en dessous, en alternance avec des oreillers à broderie ou dentelle blanche (chez Ileana V., Mãricuta V. et Anisia L.). Dans les maisons où il y a des personnes âgées, les oreillers disparaissent ; il reste seulement les taies d'oreiller. Chez Simion V. on a alterné celles qui ont des ornements floraux et celles qui sont à dentelle blanche, et chez Maria I., les taies d'oreiller ont des ornements floraux et des dentelles. Les oreillers sont des objets forts pour ce qui est de leur présence et de leurs ornements floraux (devenus traditionnels ici), et, dans le même temps, des objets faibles comme on peut le constater par le dynamisme actuel 
qui influe sur le changement des ornements. Dans les maisons où il y a des personnes âgées, les oreillers disparaissent pour ne laisser que les taies d'oreiller qui, dans ce cas, deviennent des objets faibles.

Comme nous venons de le montrer, les règles d'organisation de la ruda sont similaires dans les deux localités. Ainsi, à Sãliştea de Sus, il y avait la ruda de Maria V., née à Dragomiresti, qui a décoré la poutre comme dans son village natal, un autre village situé sur la Vallée de l'lza où la ruda suit les mêmes règles d'organisation. Mais il y a des différences par lesquelles s'affirme l'importance de l'appartenance à une communauté. Au-delà du nombre de couches (quatre pour leud et six pour Sãliştea de Sus et Dragomireşti), et au-delà des ornements (géométriques pour leud et Dragomireşti et floraux pour Sãliştea de Sus), il y a des différences concernant l'arrangement des objets sur la ruda. A leud (et Dragomireşti), les objets pendent moins dans l'espace entre la ruda et le lit qui est en dessous (où se met un autre tissu). Comme Maria V. l'explique,

La différence est la suivante : chez nous [id est Dragomireşti] l'arrangement est plus court et chez eux [id est Sãliştea de Sus] plus long. Chez nous on met des tapis sous la ruda, et à Sãliştea de Sus, on ne met rien, parce que la ruda va jusqu'au lit. [Chez nous] on met de la toile de besaces seulement pour ne pas voir le mur nu (Maria V. 2005).

Dans les maisons où il n'y a plus de ruda, celle-ci est remplacée par des tapis qui sont mis directement sur les murs et sur lesquels on dispose une icône qui est encadré par un essuie-mains brodée, comme il est possible de le voir dans les maisons de Anuta I., Ioana B., Anuta V. et Mãricuta P. Les tapis qui sont mis sur les murs, une pratique récente selon Boris Zderciuc (Zderciuc 1963: 14), sont les mêmes tapis qui se font pour la ruda et leurs ornements sont les mêmes que ceux pour la ruda.

Passer les fêtes dans la "belle salle"

Les objets qui sont sous la ruda et dans la "belle salle " suivent un ordre donné, "on ne peut pas les mettre seulement sur les murs " (Anisia L. 2001). On perpétue ainsi une tradition. Ceci serait, néanmoins, une analyse incomplète si l'on ne tentait pas de comprendre l'ensemble de la salle et de la ruda, au travers de son contexte d'utilisation et des pratiques des acteurs. 
À présent, je souhaiterais essayer de répondre à la question de savoir quand et comment les gens de Sãliştea de Sus et de Ieud utilisent la "belle pièce ", à travers les observations directes et les descriptions que les gens font pour répondre à cette question, en concentrant l'analyse sur les rituels de noces.

Une première fonction essentielle de la "salle de devant " est celle de recevoir les hôtes ou les gens de passage:

Là, dans cette pièce, des gens de toutes les catégories sociales sont entrés : prêtre, docteur, ingénieur, professeur, paysan. Ils ont été bien reçus dans cette pièce. Pour cette raison l'homme simple a essayé de la garder toujours propre, nettoyée (Gãrvilã H. 2005).

Même s'il n'y a pas de poêle, quand il y a des visiteurs, les gens en amènent un pour se chauffer. Recevoir les visiteurs dans la " belle salle ", où ils peuvent aussi dormir, leur montrer ce qu'il y a de mieux dans la maison, tout cela témoigne de l'hospitalité des gens de Maramureş, soulignant ainsi l'importance de cet espace qui prend une fonction sociale et montre le statut de son propriétaire. Les visiteurs sont associés à la fête, parce que l'habitant du Maramureş est heureux d'avoir des hôtes. Il dit que si personne ne lui rend visite, c'est qu'il n'a pas de bonne réputation (Florica P. 2001).

Il est possible de voir un transfert du familial au festif, car les membres de la famille qui n'habitent plus dans la même maison ou dans le même village sont reçus eux aussi comme les visiteurs, dans la belle salle. Si, lorsqu'ils habitaient dans la maison, il leur était interdit de dormir dans la «belle salle », il leur est désormais possible de le faire.

Ainsi, dans cette pièce est concentré tout ce qui est exclu du quotidien. Elle représente l'espace festif par excellence, où on n'entre que rarement, un espace qui reste virtuel, ouvert pour tout événement familial important.

Effectivement, dans tous les entretiens on trouve cette association de la "salle de devant" avec le temps de la fête. Les actions profanes, quotidiennes sont interdites dans cet espace, comme Viorica I. (2001) l'explique: "on ne peut pas rester là, faire quelque chose d'autre, faire à manger par exemple, ou dormir ". Comme lleana V. le déclare : " pour nous qui sommes des paysans, la salle avec la ruda est la plus festive de toute la maison, de tout ce que nous avons " (Ileana V. 2005). 
Pour cette raison, toutes les fêtes de famille et les fêtes calendaires (comme Pâques, Noël) sont célébrées dans cette salle. Les mots de Toader C. résument l'utilisation de la «belle salle " :

Quand nous avons des noces et quand nous avons des enterrements. Tu peux faire une noce là-bas. Quand une personne est morte, elle est portée dans cette pièce, où nous n'habitons pas, où il n'y a pas de feu. Nous ne faisons pas de feu si nous n'y sommes pas. Seulement pendant les fêtes. À Noël, si on fait quelque chose, on fait du feu (Toader C. 2005).

Ileana V. raconte comment après la mort de sa belle-mère, le corps de cette dernière est resté dans cette pièce:

Ma belle-mère a vécu avec nous. ... Elle vivait dans la cuisine et, quand elle est morte, elle disait " ils vont me mettre dans la maison, là où est la ruda, où c'est le plus beau ". Et c'est là qu'on l'a mise, là où était la ruda (Ileana V. 2005).

Un autre exemple est celui de Ion Simion V., qui déclare qu'au moment de son enterrement, il va être mis dans cette pièce, puisque c'est la plus festive. En guise de dernier hommage, le défunt, habillé de ses meilleurs vêtements, parfois même réalisés spécialement pour cette occasion, est veillé dans la plus belle salle de la maison.

\section{La noce dans la " belle salle"}

Mais mon enquête s'est également concentrée sur les rites de noces. La "belle salle", qui porte partout les signes de la noce, est l'espace le plus utilisé pendant ce rite de passage. Un exemple, très significatif, est le fait que Anuta D. de Ieud a mis sur la ruda son bouquet de mariée, puisqu'elle venait de se marier en 2004 (voir photo no. 4). Comme la ruda donne à voir la dot des filles, son geste devient symbolique. L'assimilation de la poutre suspendue, et les objets qui la composent, à la mariée est évidente dans le discours de Maria V., mais aussi dans celui de Mãricuta V., de Sãliştea de Sus. Elles ont fait une belle comparaison entre les vêtements de la mariée et les couches de la ruda:

La ruda se fait comme la mariée de jadis. La mariée de jadis avait des bas, puis une robe, puis de nouveaux des bas, de nouveau une robe et puis la jupe paysanne (zadia). Après tout ça, la veste de laine. Et c'était comme ça la mariée de jadis, il faut la ruda comme ça aussi. Au début il faut mettre les couvertures en laine, après les nappes. Après les nappes, les besaces, après les besaces, une nappe, après la nappe, les tapis et tout en haut, les oreillers (Mãricuta V. 2005). 


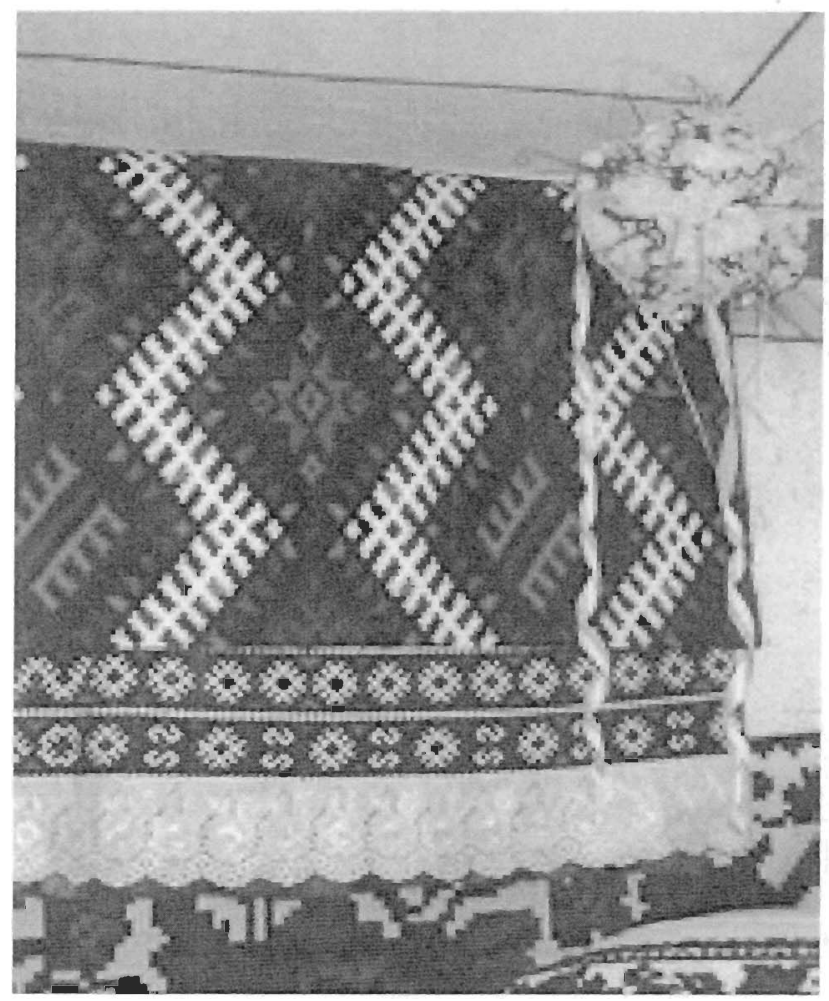

4. Le bouquet de marié sur la ruda à leud (A. Iuga, 2004).

Traditionnellement, tous les moments de la noce se passent à l'intérieur de la maison, et dans la belle salle:

Tout commence et tout finit dans la "belle salle ". Dans le jour de la noce ... tout commence le matin. Beaucoup d'agitation, beaucoup de monde. Beaucoup de fatigue. ... Et tout commence dans la " grande maison », dans la "belle salle ", qui est bien rangée pour recevoir les invités (Viorica I. 2005).

Les meubles sont déplacés dehors, il ne reste plus que la table, les banquettes et les chaises pour que les invités aient de la place. Et, bien sûr, s'il y a la ruda, cela reste sur les murs. Même aujourd'hui il y a beaucoup de personnes qui viennent seulement pour voir la mariée, ou le marié, et qui regardent et discutent aussi de la beauté et des richesses de la ruda. 
Mais tout commence le soir d'avant, quand les autres jeunes du village viennent à la maison des jeunes qui vont se marier. À la maison du marié - les jeunes garçons vont préparer "la bannière ":

Il y avait autant la coutume de coudre "la bannière " et de danser "la bannière ». Avant la noce il y avait la dance de "la bannière ". Tous les garçons aidaient: ils apportaient des perles, des foulards, des serviettes, des bandes de tous les couleurs. Et ils préparaient "la bannière" (Maria V. 2005).

Pendant ce moment il y a de la musique, des chansons, des acclamations en vers qui sont sur le même thème: la séparation du marié du groupe social des jeunes garçons.

Le jour de la noce, chez la mariée il y a la préparation de la couronne de la mariée, préparée par les amies de la mariée. Il y a aussi de la musique, des acclamations :

Toutes les acclamations sont sur le même thème : la mariée qui pleure, la mariée qui va quitter sa famille, ses amis. Toutes les acclamations sont assez tristes. Il n'y a pas beaucoup de joie dans ce moment. Beaucoup de femmes pleurent, peut-être se rappellent-elles leur expérience (Viorica 1., 2005).

Un des moments les plus importants de la noce c'est " la demande des pardons", marquant le rite de passage des noces (voir photo no. 5). Les mariés sont chacun dans leur maison avec toute leur famille présente dans la " belle salle ». Le marié (ou la mariée) est assis derrière la table (sur laquelle il y a une gimblette décorée avec de basilic et des fils rouges), et à ses cotés il y a les parrains. Il/elle demande pardon, premièrement à ses parents, pour les erreurs qu'il/elle avait fait pendant son enfance et pendant le temps quand il/elle vivait avec ses parents. C'est un moment nécessaire pour recevoir le pardon des parents et leur bénédiction. Ensuite, il/elle demande pardon aux autres membres de sa famille. Toute cette scène, telle qu'observée en 2006 à la noce de Nicolae M., se passe dans la belle salle.

Il y a cinquante ans, après la cérémonie religieuse, la fête de la noce se passait dans la maison : la mariée allait avec ses invités et sa famille chez ses parents, et le marié, chez lui :

À la noce, chacun à sa maison. La famille de la mariée chez elle, celle du marié chez lui. Ils mangeaient, ils dansaient, et puis, à un moment, vers le matin, quelques personnes [de la famille du marié] étaient choisies pour aller chercher la mariée (Maria V. 2005). 


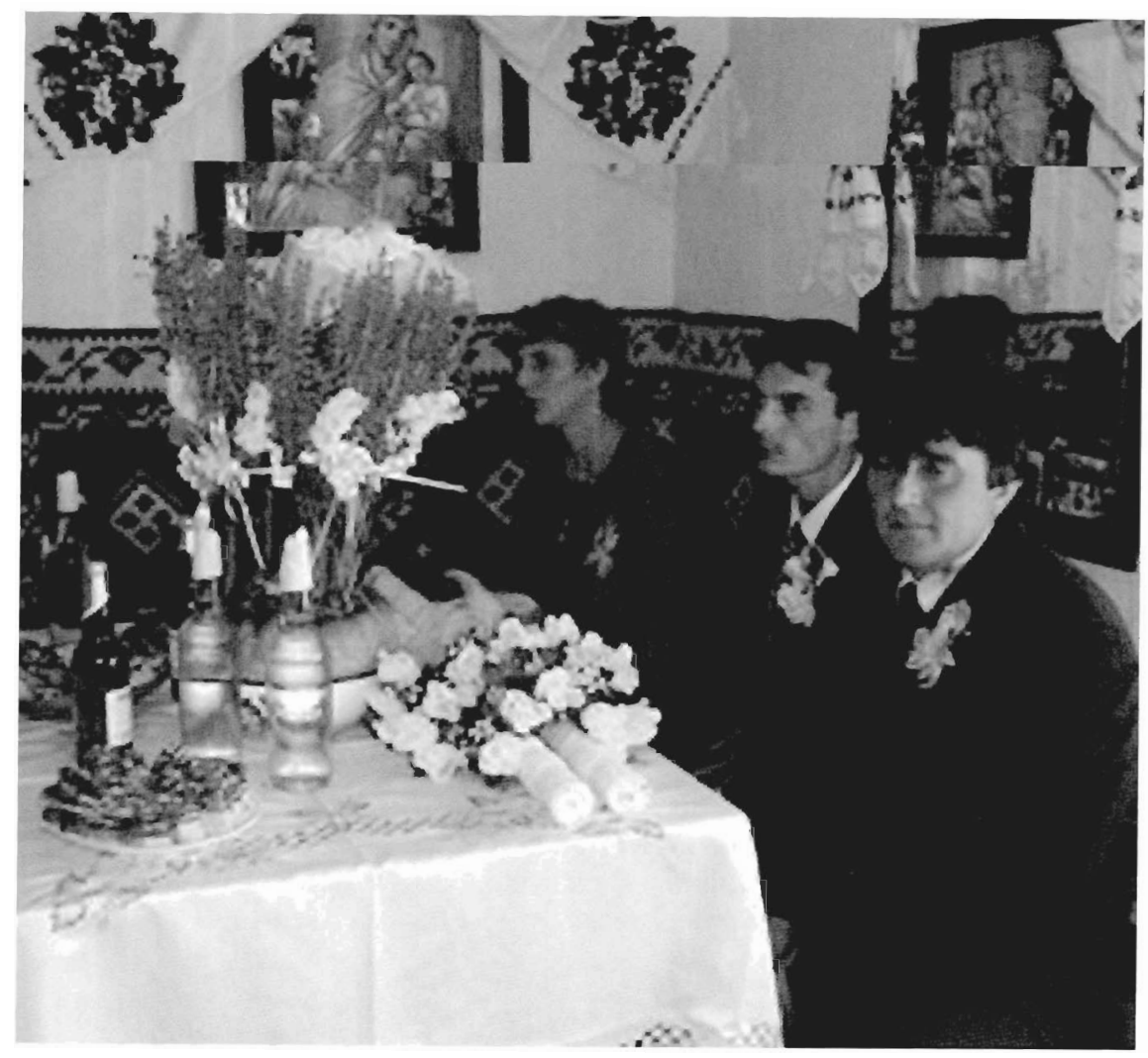

5. La demande des pardons dans la "belle salle" (A. Iuga, 2004).

Quand la mariée vient chez le marié, les deux sont assis dans la "belle salle ", la plus belle salle. La noce se déroulait dans toute la maison, mais les mariés se trouvaient dans la "belle salle».

Quand on organisait les noces dans la maison, il n'y avait pas assez d'espace pour tous dans cette salle. Et si tu n'avais pas une place là-bas ... Les gens racontaient : J'étais à la noce, mais j'ai eu une place dans l'autre salle! Et ça valait beaucoup (Maria C. 2006).

La place où les invités étaient assis pendant la noce exprimait leur position sociale par rapport à la famille qui fêtait la noce. Pour les personnes âgées, la meilleure place était en dessous de la ruda ; 
Les personnes âgées étaient en dessous de la ruda, puisqu'ils ne dansaient pas. Les jeunes étaient assis vers l'intérieur de la salle, et à côté étaient assises les personnes âgées qui aimaient voir les jeunes de la famille danser. En dessous de la ruda c'était une place d'honneur pour les vieux (Maria V. 2005).

La dimension de la salle est donc d'une grande importance. Une bonne position sociale impliquait des obligations normatives plus grandes, car la famille était plus grande et devait donner l'exemple dans la communauté. Pour ce moment-là, si tu avais une grande famille, il faillait que tu construises une maison avec une grande "belle salle". Gavrilã H. raconte comment, pour les noces de sa fille, il est allé parler avec son voisin qui avait une "belle salle " plus grande, pour qu'il y fasse la noce :

En 1983, quand j'ai fait les noces de ma fille, j'ai su que les invités n'auraient pas assez de place dans ma belle salle, parce que j'avais une pièce de cinq mètres sur cinq. J'ai su qu'ils n'allaient pas tous entrer parce que j'avais invité mes collègues du musée et les villageois, et alors j'ai parlé avec un homme qui habite ici, derrière ma maison. C'est un bon voisin, il a une grande pièce, de six mètres sur six. "Stefane, me laisserais-tu [ta belle salle], je vais te payer s'il faut". "]e n'ai pas besoin d'argent, tu peux faire le repas de noce ici ». Et j'ai fait la noce chez mon voisin (Gavrilã H. 2005).

Ces dernières années, comme les noces sont de plus en plus grandes, puisque des personnes de l'extérieur de la communauté y participent aussi, il y a de plus en plus d'invités, et les invités n'ont plus assez de place dans une pièce. Ainsi, les noces ont lieu maintenant dans le Foyer Culturel, situé au centre du village. Même là les villageois mettent des tissus et décorent les murs avec des tapis.

La fin de la noce, un autre moment important de passage, se déroule toujours dans la "belle salle ", même aujourd'hui, quand les noces ne passent plus entièrement dans la maison.

Vers cinq heures, quand il n'y a plus personne, les mariées vont à la maison. Ils montent dans une voiture et vont à la maison du marié. Dans la belle salle, là-bas, ils sont reçus par la mère du marié. C'est là que la belle-mère transforme la mariée en épouse (Viorica I. 2005).

C'est la pratique de mettre le foulard sur la tête de la mariée : le passage symbolique d'une catégorie sociale (jeune fille non mariée), à une autre (jeune femme mariée). 


\section{Conclusion}

Ieud et Sãliştea de Sus sont des communautés qui fonctionnent selon les règles "traditionnelles» qui " ont toujours fait partie de la conscience de soi [des gens] »(Kligman 1998: 11), étant incluses dans les pratiques quotidiennes de vie. Il y a des coutumes bien préservées. Il y a toujours des maisons et des églises qui sont construites en hois, outre celles qui sont anciennes et qui font que le Maramureş est connu dans le monde entier. Beaucoup de personnes (plus de femmes que d'hommes) portent encore des vêtements qui suivent des règles traditionnelles, et ce costume devient une marque d'appartenance, une preuve matérielle et une revendication qu'ils appartiennent à la région du Maramureş : "On peut reconnaître les gens du Maramureş par les vêtements" et, si ça se perdait, "on ne serait plus du Maramureş, personne ne pourrait plus nous reconnaître" (Anisia L. 2001)). Les maisons sont ornées avec des tissus faits par les femmes elles-mêmes: dans toutes les salles il est possible de trouver des objets faits à la main.

Néanmoins, il y a des signes de changement partout, non seulement dans la "belle salle", mais dans toutes les pratiques culturelles. La plupart des coutumes se sont adaptées aux changements économiques et sociaux. L'architecture des villages a beaucoup changé dans les trente dernières années, et l'on construit désormais des maisons en briques et en béton avec des étages parfois en bois. Les vêtements ont changé et il y a des innovations dans les pièces vestimentaires traditionnelles, influencées par d'autres modes. Les objets qui décorent la maison sont de plus en plus modernes : il y a divers appareils électroniques qui sont achetés le plus tôt possible (toujours selon le désir d'être à la mode), et qui témoignent d'une "tendance à redéfinir le soi à travers les biens possédés " (Kligman 1998: 11).

Du point du vue de la culture matérielle, de sa signification et de son usage, il y a un mélange entre la tradition et l'innovation, lié à l'affirmation de l'appartenance d'un individu à une communauté et à celle de son statut social.

Ainsi, tout passage vers une autre règle communautaire dépend du fort accrochement aux traditions et de l'ouverture vers l'innovation, en lien avec le contexte d'utilisation, de création et de leur signification. Par exemple, les femmes tissent et font une "belle pièce " pour se conformer aux règles de leur communauté ; elles font leur propre dot, mais elles le font aussi pour affirmer leur propre individualité, car chaque 
"salle de devant " est unique en son genre, et elles tissent surtout pour affirmer leur position sociale, mise en valeur surtout pendant les événements qui se déroulent dans cet espace. La pièce qui a été le sujet de cette analyse devient, ainsi, un discours important sur le soi ; elle communique l'attachement à la fois aux traditions, et à la modernité.

La dynamique de la "belle salle " est éloquente quant à la dynamique de la culture des gens pour lesquels elle est toujours importante. $\mathrm{La}$ " belle salle » est un aspect culturel spécifique et toujours vivant, qui n'est pas figé dans un modèle unique, et qui a encore de la valeur et de la signification pour les habitants de leud et Saliştea de Sus. La " bonne salle " est toujours d'actualité, elle s'adapte à des nécessités nouvelles, elle change, mais dans le même temps, elle reste immuable. 


\section{Références}

Bobu Florescu, Florea et Paul Petrescu, 1969, Arta popularã româneascã. Bucarest, Academia Republicii Socialiste Române.

Cristescu Golopentia, Stefania, 2002, Gospodãria în credintele şi riturile magice ale femeilor din Drãguş (Fãgãraş). Bucarest, Paideia.

Dima, Alexandru, 1971, Arta popularã şi relatiile ei. Bucarest, Minerva.

Filipaşcu, Alexandru, 1954, Voevodatul Maramureşului. Originea, structura şi tendinpele lui. Sibiu, Astra Culturalã.

Hall, Edward T., 1971, La dimension cachée. Paris, Seuil.

Kligman, Gail, 1998, Nunta mortului. Ritual, poeticã și culturã popularã in Transilvania. Iași, Polirom.

Nistor, Francisc, 1980, Arta lemnului in Maramureş. Centrul judetean de îndrumare a creapiei populare şi a mişcãrii artistice de masã, Baia Mare.

Pleşu, Andrei, 1986, Ochiul şi lucrurile. Bucarest, Meridiane.

Popescu, Ioana, 2002, Foloasele privirii. Bucarest, Paideia.

Stoica, Georgeta et Doagã, Aurelia, 1977, Interioare românești. Desãturi şi cusãturi decorative. Bucarest, Albatros.

Van Gennep, Arnold, 1996, Riturile de trecere. Iaşi, Polirom.

Vlad, Maria, 2002, "Obiceiuri şi cretinte din Sãliştea de Sus ». Memoria ethnologica 4-5.

Zderciuc, Boris, 1963, Covorul maramureşean. Bucarest, Meridiane.

Zderciuc, Boris, Paul Petrescu et Tancred Bãnãteanu, 1964, Arta popularã in România. Bucarest, Meridiane.

\section{Entrevues}

Anisia L., 47 ans, Sãliştea de Sus, 18 avril 2001

Anuta D., 23 ans, leud, 2 février 2005

Anuta 1., 57 ans, Sãliştea de Sus, 23 janvier 2005

Anuta V., 60 ans, Sãliştea de Sus, 20 janvier 2005

Florica P., 38 ans, Sãliştea de Sus, 19 avril $2001 ; 19$ janvier 2005.

Gavrila H., 67 ans, sculpteur en bois, retraité, leud, 1 février 2005

Ileana V., 43 ans, Sãliştea de Sus, 20 janvier 2005

Ioana B., 66 ans, Sãliştea de Sus, 21 janvier 2005

Ioana H., 62 ans, Ieud, 1 février 2005

Ion Simion V., 70 ans, retraité, Sãliştea de Sus : 18 avril $2001 ; 21$ janvier 2005

Irina C., 75 ans, leud, 2 février 2005

Maria C., 50 ans, Sãliştea de Sus, 26 décembre 2006

Maria Ch., 46 ans, Sãliştea de Sus, 20 janvier 2005

Maria V., 52 ans, institutrice, Sãliştea de Sus, 22 janvier 2005 
Maria V., 53 ans, institutrice, Sãliştea de Sus, 18 décembre 2005 Mãricuta C., 36 ans, leud, 2 février 2005

Mãricuta P., 30 ans, Sãliştea de Sus, 22 janvier 2005

Mãricuta V., 64 ans, Sãliştea de Sus, 22 janvier 2005

Simion I., 52 ans, professeur d'histoire, Sãliştea de Sus, 19 avril 2001

Toader C., 78 ans, leud., 2 février 2005

Viorica I., 22 ans, étudiante de Cluj-Napoca, Sãliştea de Sus, 1 avril 2001

Viorica I., 26 ans, juriste, Sãliştea de Sus, 20 décembre 2005 